Sādhanā Vol. 36, Part 2, April 2011, pp. 153-165. (C) Indian Academy of Sciences

\title{
Land cover classification using reformed fuzzy C-means
}

\author{
B SOWMYA* and B SHEELARANI
}

Department of Electronics and Communication Engineering Jeppiaar Nagar, Sathyabama University, Old Mamallapuram Road, Chennai, Tamilnadu 600 119, India

e-mail: bsowya@yahoo.com

MS received 25 June 2009; revised 11 January 2011; accepted 23 February 2011

\begin{abstract}
This paper explains the task of land cover classification using reformed fuzzy $\mathrm{C}$ means. Clustering is the assignment of objects into groups called clusters so that objects from the same cluster are more similar to each other than objects from different clusters. The most basic attribute for clustering of an image is its luminance amplitude for a monochrome image and colour components for a colour image. Since there are more than 16 million colours available in any given image and it is difficult to analyse the image on all of its colours, the likely colours are grouped together by clustering techniques. For that purpose reformed fuzzy $\mathrm{C}$ means algorithm has been used. The segmented images are compared using image quality metrics. The image quality metrics used are peak signal to noise ratio (PSNR), error image and compression ratio. The time taken for image segmentation is also used as a comparison parameter. The techniques have been applied to classify the land cover.
\end{abstract}

Keywords. Land cover; FCM; RFCM; clustering; compression ratio; PSNR; kappa coefficient.

\section{Introduction}

Land cover refers to features of land surface. These can be natural, semi-natural, managed or totally man-made. They are directly observable. The main reason for producing land cover maps is to give us a clear idea of the stock and state of our natural and built resources. A land cover classification, is an essential component in developing a responsible attitude to environmental management. Land cover is distinct from land use despite the two terms often being used interchangeably. Land use is a description of how people utilize the land and socio-economic activity-urban and agricultural land uses are two of the most commonly recognised high-level classes of use. At any one point or place, there may be multiple and alternate land uses, the specification of which may have a political dimension. Land cover classifications are essential inputs to environmental and land use planning at local, regional, and national levels. This paper uses segmentation based on unsupervised clustering techniques for classification of land cover.

*For correspondence 
Partitioning of an image into several constituent components is called image segmentation. Segmentation is an important part of practically any automated image recognition system, because it is at this moment that one extracts the interesting objects, for further processing such as description or recognition. Segmentation of an image is in practice the classification of each image pixel to one of the image parts. So image segmentation and classification of its pixels into groups are one and the same, the words clustering and segmentation are used alternatively.

Image segmentation has been the subject of considerable research activity over the last three decades. Many unsupervised algorithms have been developed for segmenting gray scale images. In computer vision literature, various methods dealing with segmentation and feature extraction are discussed, which can be broadly grouped into region-based techniques, edge-based techniques, hybrid methods which combine edge and region methods, and so on. However, because of the variety and complexity of images, robust and efficient segmentation algorithm on colour images are still a very challenging task and fully automatic segmentation procedures are far from satisfying in practical situations As colours convey more information than the intensities, this paper explains the task of classifying each pixel in an image into one of a discrete level of colour classes using different fuzzy clustering techniques. Colour image segmentation is done by mapping of a pixel into a point in an $n$-dimensional feature space, defined by the vector of its feature values. The problem is then reduced to partitioning the feature space into separate clusters, which is a general pattern recognition problem.

The paper is organized as follows. The earlier related works and their limitations are discussed in section 2. In section 3, different clustering techniques and classification method are discussed. In section 4, composite satellite image and colour components of different surface features are dealt with. In section 5, important quality measurement techniques like PSNR, error image are discussed. Evaluation of results and land cover classification are given in section 6. Concluding remarks are given in section 7.

\section{Related work}

Fuzzy C-means is a method of clustering which allows one data to belong to two or more clusters. This method was developed by Dunn (1973) and improved by Bezdek (1981) and is frequently used in image segmentation and pattern recognition. The main disadvantage of FCM is that the sum of membership values of a data point in all the clusters must be one and so the algorithm has difficulty in handling outlier points (Cox 2005). Frank Klawonn \& Annette Keller (1997) have proposed a modified $\mathrm{C}$-means algorithm with changed distance function which is the dot product instead of the conventional Euclidean distance. This method is used for identifying clusters of new shapes. An additional term is injected into the objective function to constrain the behaviour of membership functions with the neighbourhood effect by Lei Jiang \& Wenhui Yang (2003). Krishnapuram \& Keller (1996) have proposed a fuzzy-possibilistic C-means (FPCM) model and algorithm that generated both membership and typicality values when clustering unlabelled data. FPCM constrains the typicality values so that the sum of over all data points of typicality to a cluster is one. The row sum constraint produces unrealistic typicality values for large data sets. Later they have modified it by a new model called possibilistic-fuzzy C-means (PFCM) model. PFCM produces memberships and possibilities simultaneously, along with the usual point prototypes or cluster centers for each cluster (Pal et al 2005). Both FPCM and PFCM require some boot strap method for initialization of weights.

FCM is the most popular fuzzy clustering algorithm. Researchers have used this algorithm for different applications. Problems related to remote sensing data clustering for both supervised 
and unsupervised classification can be solved by FCM. Wang used supervised FCM to classify the Landsat MSS and TM data with seven land cover classes (Wang 1990). The results were compared with the maximum likelihood classification. The conclusion was that higher classification accuracy could be achieved while using fuzzy classification approach. Foody has evaluated the performance of FCM and fuzzy neuron network for land cover classification from airborne thematic mapper (ATM) data. He studied the effect of different fuzzy parameter (m) values for the same dataset and found that $m=2.0$ gives most accurate fuzzy classification output for many cases. He concluded that the fuzzy classification technique provides more appropriate results in land cover mapping than hard classification techniques (Foody 1996). Bastin compared FCM, linear mixture modeling and maximum likelihood classifier for unmixing coarse pixels present in aggregated Landsat TM data. In absence of ground truth the original TM data were used as reference map and aggregated the image using mean and cubic filter with different kernel size. The author concluded that FCM gives the best prediction of sub-pixel land cover classes for the aggregated TM image at different scale (Bastin 1997).

Foody \& Zhang (1998) used fuzzy C means algorithm for sub-urban land cover mapping from SPOT HRV and Landsat TM data. They found that the classification results could be improved significantly while using fuzzy classification and evaluation approaches (Foody \& Zhang 1998). Ibrahim et al (2005) compared different fuzzy classification techniques to generate accurate land cover maps in presence of uncertainties. In their study they concluded that possibilistic means gives the highest accuracy in land cover mapping, followed by the FCM technique (Ibrahim et al 2005).

\section{Fuzzy clustering algorithms and classification method}

In real applications very often no sharp boundary between clusters so that fuzzy clustering is often better suited for the data. Membership degrees between zero and one are used in fuzzy clustering instead of crisp assignments of the data to clusters.

The resulting data partition improves data understanding and reveals its internal structure. Partition clustering algorithms divide up a data set into clusters or classes, where similar data objects are assigned to the same cluster whereas dissimilar data objects should belong to different clusters.

Areas of application of fuzzy cluster analysis include data analysis, pattern recognition, and image segmentation. The detection of special geometrical shapes like circles and ellipses can be achieved by so-called shell clustering algorithms.

\subsection{Fuzzy C means}

The most prominent algorithm is the FCM or fuzzy $\mathrm{C}$ means algorithm. The fuzzy $\mathrm{C}$ means algorithm was proposed as an improvement of the classic hard C-means clustering algorithm. The FCM algorithm receives the data or the sample space, an $n \times m$ matrix where $n$ is the number of data and $m$ is the number of parameters. The number of clusters $c$, the assumption partition matrix $U$, the convergence value $E$ all must be given to the algorithm. The assumption partition matrix has $c$ number of rows and $n$ number of columns and contains values from 0 to 1 .

The sum of every column has to be 1 . The first step is to calculate the cluster centers. This is a matrix $v$ of dimension $c$ rows with $m$ columns. The second step is to calculate the distance matrix $D$. The distance matrix constitutes the Euclidean distance between every pixel and every 
cluster center. This is a matrix with $c$ rows and $n$ columns. From the distance matrix the partition matrix $U$ is calculated.

If the difference between the initial partition matrix and the calculated partition matrix is greater than the convergence value then the entire process from calculating the cluster centers to the final partition matrix is repeated. The final partition matrix is taken and is used for reconstructing the image. Let us assume as a fuzzy C-Means Functional,

$$
J_{m}(U, Y)=\sum_{k=1}^{n} \sum_{j=1}^{c} u_{j k}^{m} E_{j}\left(x_{k}\right),
$$

where, $\mathrm{X}=\left\{x_{\mathrm{k}} \mid \mathrm{k} \in[1, \mathrm{n}]\right\}$ is the training set containing $n$ unlabelled samples, $\mathrm{Y}=\left\{y_{\mathrm{j}} \mid \mathrm{j} \in\right.$ $[1, \mathrm{c}]\}$ is the set of centers of clusters, $E_{\mathrm{j}}\left(x_{\mathrm{k}}\right)$ is a dissimilarity measure (distance or cost) between the sample $x_{\mathrm{k}}$ and the center $y_{\mathrm{j}}$ of a specific cluster $j, U=\left[u_{j k}\right]$ is the $c \times n$ fuzzy c-partition matrix, containing the membership values of all samples in all clusters and $m \in(1, \infty$ is a control parameter of fuzziness.

The clustering problem can be defined as the minimization of $J_{m}$ with respect to $Y$, under the probabilistic constraint:

$$
\sum_{j=1}^{c}\left(u_{j k}\right)=1 .
$$

The fuzzy C-means (FCM) algorithm consists in the iteration of the following formulas. The cluster centers are calculated by,

$$
y_{j}=\frac{\sum_{k=1}^{n}\left(u_{j k}\right)^{m} x_{k}}{\sum_{k=1}^{n}\left(u_{j k}\right)^{m}} \forall j
$$

and the membership values are determined by,

$$
\begin{gathered}
\left.u_{j k}=\left(\sum_{l=1}^{c}\left(\frac{E_{j}\left(x_{k}\right)}{E_{l}\left(x_{k}\right)}\right)^{2 / m-1}\right)^{-1} \text { if } E_{j}\left(x_{k}\right)\right\rangle 0 \forall j, k \\
1 \text { if } E_{j}\left(x_{k}\right)=0 \text { and } u_{j k}=0 \forall l \neq j k,
\end{gathered}
$$

whereas, in the case of the Euclidean space

$$
E_{j}=\left\|x_{k}-y_{j}\right\|^{2}
$$

It is worth noting that if one chooses $m=1$ the fuzzy C-means functional $J_{m}$

Eq. 1 reduces to the expectation of the global error

$$
\langle E\rangle=\sum_{k=1}^{n} \sum_{j=1}^{c} u_{j k} E_{j}\left(x_{k}\right),
$$

and the FCM algorithm becomes the classic hard C-means algorithm. 


\subsection{Possibilistic fuzzy C means}

In the possibilistic approach to clustering the membership function or the degree of typicality of a point in a fuzzy set (or cluster) is assumed to be absolute. In other words, the degree of typicality does not depend on the membership values of the same point in other clusters contained in the problem domain. By contrast, many clustering approaches impose a probabilistic constraint, according to which the sum of the membership values of a point in all the clusters must be equal to one. As a consequence, HCM, FCM and many other clustering methods assuming the probabilistic constraint cannot generate membership functions whose values can be interpreted as degrees of typicality.

PCM algorithm avoids the assumption of the probabilistic constraint. The PCM is based on the relaxation of the probabilistic constraint in order to interpret in a possibilistic sense the membership function or degree of typicality. The possibilistic C-means algorithm I is based on a modification of the objective function of FCM. In this case, one must supply the values of some parameters such as the fuzzifier parameter, and others regulating the weight of the spread of membership functions. The possibilistic C-means algorithm II is based on modification of the cost function of the HCM instead of the FCM in order to avoid, in this way, the determination of the fuzzifier parameter. The objective function of the PCM-II contains two terms as shown below.

$$
J(U, Y)=\sum_{k=1}^{n} \sum_{j=1}^{c} u_{j k} E_{j}\left(x_{k}\right)+\sum_{j=1}^{c} \rho_{j} \sum_{k=1}^{n}\left(u_{j k} \ln u_{j k}-u_{j k}\right) .
$$

The first one is the objective function of the HCM, while the second is a regularizing term, forcing the values $u_{j k}$ to be greatest as possible, in order that points with a high degree of typicality with respect to a cluster may have high $u_{j k}$ values, and points not very representative may have low $u_{j k}$ values in all clusters. In the above equation $\mathrm{E}_{\mathrm{j}}\left(x_{\mathrm{k}}\right)=\left|x_{\mathrm{k}}-y_{\mathrm{j}}\right|^{2}$ is the square of the Euclidean distance, and the parameter $\mathrm{P}_{j}$ depends on the distribution of point in the $\mathrm{j}^{\text {th }}$ cluster.

$$
\begin{gathered}
y_{j}=\frac{\sum_{k=1}^{n}\left(u_{j k}\right) x_{k}}{\sum_{k=1}^{n}\left(u_{j k}\right)} \quad \forall j \\
u_{j k}=\exp \left\{-\frac{E_{j}\left(x_{k}\right)}{\rho_{j}}\right\} \quad \forall j, k .
\end{gathered}
$$

This theorem provides the conditions needed in order to minimize the cost function. The above two equations can be interpreted as formulas for recalculating the membership functions and the cluster centers. A bootstrap clustering algorithm is anyway needed before starting PCM in order to obtain an initial distribution of prototypes in the feature space and to estimate some parameters used in the algorithm. By considering an FCM bootstrap for the PCM, the following definition of $\mathrm{P}_{\mathrm{j}}$ can be used.

$$
\rho_{j}=K \frac{\sum_{k=1}^{n}\left(u_{j k}\right)^{m} E_{j}\left(x_{k}\right)}{\sum_{k=1}^{n}\left(u_{j k}\right)^{m}},
$$


where, $m$ is the fuzzifier parameter used by the FCM, and $K$ is a proportional parameter. This definition makes $\rho_{j}$ proportional to the mean value of the intracluster distance, and critically depends on the choice of $K$.

\subsection{Reformed fuzzy $C$ means}

For reformed fuzzy $\mathrm{C}$ means a neighbourhood influence parameter $\gamma$ at each pixel is calculated. It is determined by convolving original gray scale image with a template of $3 \times 3$ matrix of ones. The probabilistic constraint is removed by equating sum of membership function in a cluster to $n$.

$$
\sum_{j=1}^{c}\left(u_{j k}\right)=n .
$$

The distance measure is modified by the neighbourhood influence parameter $\gamma$. The distance measure is

$$
E_{j}=\left\|x_{k}-y_{j}\right\|^{2} e^{-\gamma k} .
$$

The membership values are determined by,

$$
u_{j k}=\left\{\begin{array}{c}
\left.n *\left(\sum_{l=1}^{c}\left(Z_{j}\left(x_{k}\right) E_{j}\left(x_{k}\right)\right)^{2 / m-1}\right)^{-1} \text { if } E_{j}\left(x_{k}\right)\right\rangle 0 \forall j, k \\
1 \text { if } E_{j}\left(x_{k}\right)=0 \text { and } u_{j k}=0 \forall l \neq j k,
\end{array}\right\}
$$

where $Z_{j}$ is,

$$
z_{j}=\sum_{k=1}^{c} \frac{1}{E_{k}} .
$$

\subsection{Classification method}

The satellite image pixels are classified by maximum membership method. The partition matrix gives the extent to which each pixel belongs to different clusters. From the partition matrix, the optimal cluster to which the pixel maximum belongs to is selected. For each pixel, the row number of largest element in each column of the partition matrix is found and the pixel is assigned the cluster center value, corresponding to that row. In this way, each pixel is assigned, a cluster center value determined by the clustering algorithm.

\section{Geocover (composite landsat 7 satellite) image and surface features}

Earth observation satellites are satellites specifically designed to observe Earth from orbit. These satellites are used for environmental monitoring, meteorology, map making, etc. Satellites observe the Earth with different filters or bands.

The Landsat satellites have been launched to observe the Earth, and the data collected have been used to study the Earth's environment, resources, and natural and man-made changes on the Earth's surface. Landsat 7 which has been launched in April 15, 1999 carries an enhanced thematic mapper plus (https://zulu.ssc.nasa.gov/mrsid/tutorial/Landsat\%20Tutorial-V1.html). The spectral sensitivity of Landsat 7 bands are listed in table 1. 
Table 1. Spectral sensitivity of landsat 7 bands.

\begin{tabular}{ll}
\hline Band & \multicolumn{1}{c}{ Spectral sensitivity } \\
\hline 1 & $.45-.52 \mu \mathrm{m}$ blue \\
2 & $.53-.61 \mu \mathrm{m}$ green \\
3 & $.63-.69 \mu \mathrm{m}$ red \\
4 & $.75-.9 \mu \mathrm{m}$ Near IR \\
5 & $1.55-1.75 \mu \mathrm{m}$ Short Wave IR \\
6 & $10.4-12.5 \mu \mathrm{m}$ Thermal IR \\
7 & $2.1-2.35 \mu \mathrm{m}$ Short Wave IR \\
8 & $.52-.9 \mu \mathrm{m}$ Panchromatic \\
\hline
\end{tabular}

The Band 2: $0.52-0.60 \mu \mathrm{m}$ (green) corresponds to the green reflectance of healthy vegetation and is spanning the region between the blue and red chlorophyll absorption bands and Band 3: $0.63-0.69 \mu \mathrm{m}$ (red) chlorophyll absorption band of healthy green vegetation is one of the most important bands for vegetation discrimination and Band 4: 0.76-0.90 $\mu \mathrm{m}$ (near infrared) band is especially responsive to the amount of vegetation biomass present in a scene. It is useful for identification of vegetation types, and emphasizes soil-crop and land-water contrasts. A false colour composite was compiled using bands 2, 3 and 4. This composite image was used in our work for this band combination. Based on the technical expertise the surface features were identified from the tonal variations that are given in table 2 .

\section{Quality measures used for image segmentation and land cover classification}

The quality measures used for evaluating the image segmentation are peak signal to noise ratio, compression ratio and execution time. Kappa coefficient is used for evaluating the land cover classification.

\subsection{Peak signal to noise ratio}

Signal-to-noise (SNR) measures are estimates of the quality of a reconstructed image compared with an original image. The basic idea is to compute a single number that reflects the quality of the reconstructed image. Reconstructed images with higher metrics are judged better. In fact, traditional SNR measures do not equate with human subjective perception. Several research groups are working on perceptual measures, but for now signal-to-noise measures are used because they are easier to compute. Also, to be noted that higher measures do not always mean better quality.

The actual metric that is computed in this work is the peak signal-to-reconstructed image measure, which is called PSNR. Assume a source image $f(i, j)$ is given that contains $M$ by $N$ pixels and a reconstructed image $\mathrm{F}(\mathrm{i}, \mathrm{j})$ where $\mathrm{F}$ is reconstructed by decoding the encoded version

Table 2. Surface features and colour components.

\begin{tabular}{ll}
\hline Surface feature & \multicolumn{1}{c}{ Colour component } \\
\hline Forests and Vegetation & Shades of green \\
Water & Black to dark blue \\
Urban areas & Lavender \\
Bare soil & Magenta, lavender, or pale pink \\
\hline
\end{tabular}




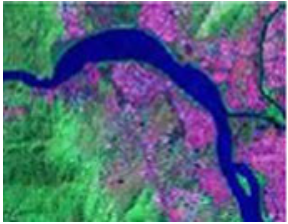

Mumbai, India

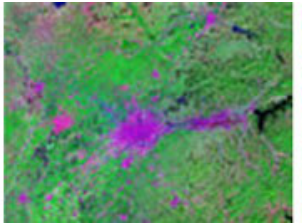

Shenyang, China

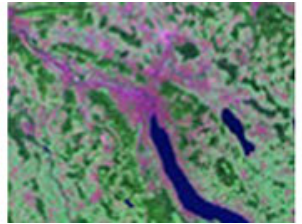

Zurich, Switzerland

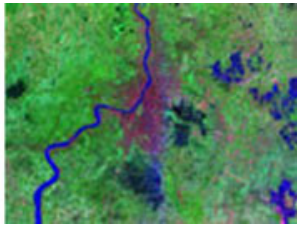

Kolkatta, India

Figure 1. Landcover images.

of $f(i, j)$. Error metrics are computed on the luminance signal only so the pixel values $f(i, j)$ range between black (0) and white (255).

First the mean absolute error (MAE) of the reconstructed image is computed as follows

$$
M A E=\frac{1}{M N} \sum_{i=1}^{M} \sum_{j=1}^{N}|F(i, j)-f(i, j)| .
$$

The summation is over all pixels. PSNR in decibels $(\mathrm{dB})$ is computed by using

$$
\mathrm{PSNR}=20 \log _{10}\left(255^{2} / \mathrm{MAE}\right) .
$$

Typical PSNR values range between 20 and 40. They are usually reported to two decimal points (e.g., 25.47). The actual value is not meaningful, but the comparison between two values for different reconstructed images gives one measure of quality. The MPEG committee used an informal threshold of $0.5 \mathrm{~dB}$ PSNR to decide whether to incorporate a coding optimization because they believed that an improvement of that magnitude would be visible. Some definitions of PSNR use 255/RMAE rather than 2552/MAE. Either formulation will work because we are interested in the relative comparison, not the absolute values. In our assignments we used the definition given above.

\subsection{Error image}

The other important technique for displaying errors is to construct an error image, which shows the pixel-by-pixel errors. The simplest computation of this image is to create an image by taking the difference between the reconstructed and original pixels. These images are hard to see because zero difference is black and most errors are small numbers, which are shades of black. The typical construction of the error image multiples the difference by a constant to increase the visible difference and translates the entire image to a gray level. The computation is

$$
E(i, j)=2|F(i, j)-f(i, j)|+128 .
$$
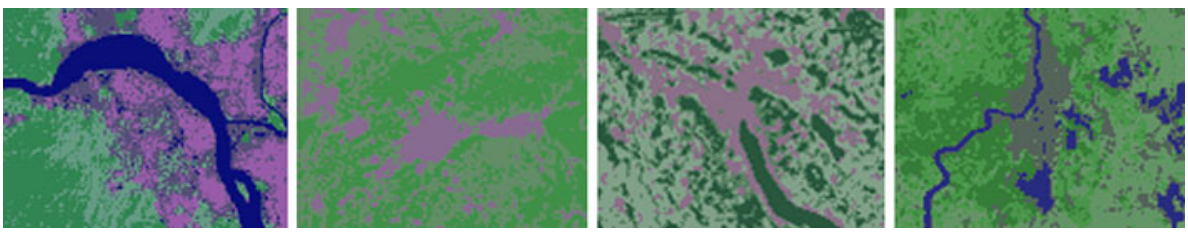

Figure 2. Classified by FCM. 

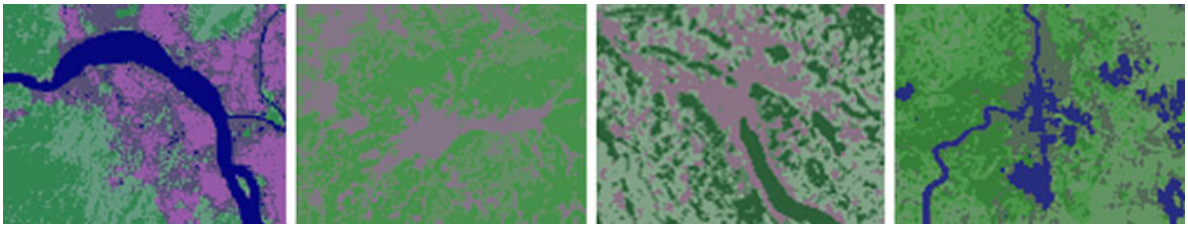

Figure 3. Classified by PFCM.

The constant 2 or the translation 128 can be adjusted to change the image. Some people use white (255) to signify no error and difference from white as an error which means that darker pixels are bigger errors.

\subsection{Compression ratio}

Data compression is the process of reducing the data required to represent information. It removes redundant and non essential data. Image segmentation can be thought of as a form of data compression, where a large number of data are converted into a small number of representative prototypes or clusters. Compression ratio is the ratio of number of bits represented by $\mathrm{n}_{1}$ required to represent the uncompressed original image and that of the image compressed $n_{2}$.

$$
\mathrm{C}_{\mathrm{R}}=\frac{\mathrm{n}_{1}}{\mathrm{n}_{2}} .
$$

If the compression ratio is more then the data required to represent the image is less. But it results in loss of data.

\subsection{Kappa coefficient}

When two binary variables are the measure of the same thing, Cohen's Kappa or Kappa Coefficient can be used as a measure of agreement between the two variables. If one variable is assumed to be the correct measure then Kappa coefficient will be the measure of correctness of the second one.

$$
K=\frac{\operatorname{Pr}(a)-\operatorname{Pr}(e)}{1-\operatorname{Pr}(e)},
$$

where $\operatorname{Pr}(a)$ is the relative observed agreement among variables, and $\operatorname{Pr}(e)$ is the hypothetical probability of chance agreement, using the observed data to calculate the probabilities of each variable randomly saying each category. If the variables are in complete agreement then $\mathrm{K}=1$. If there is no agreement among the variables then $\mathrm{K} \leq 0$.
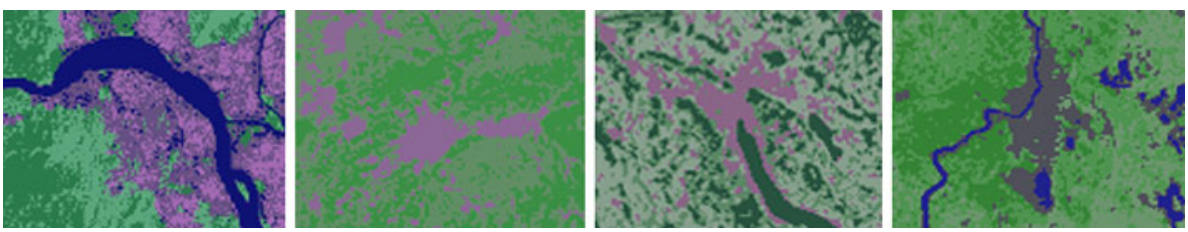

Figure 4. Classified by RFCM. 


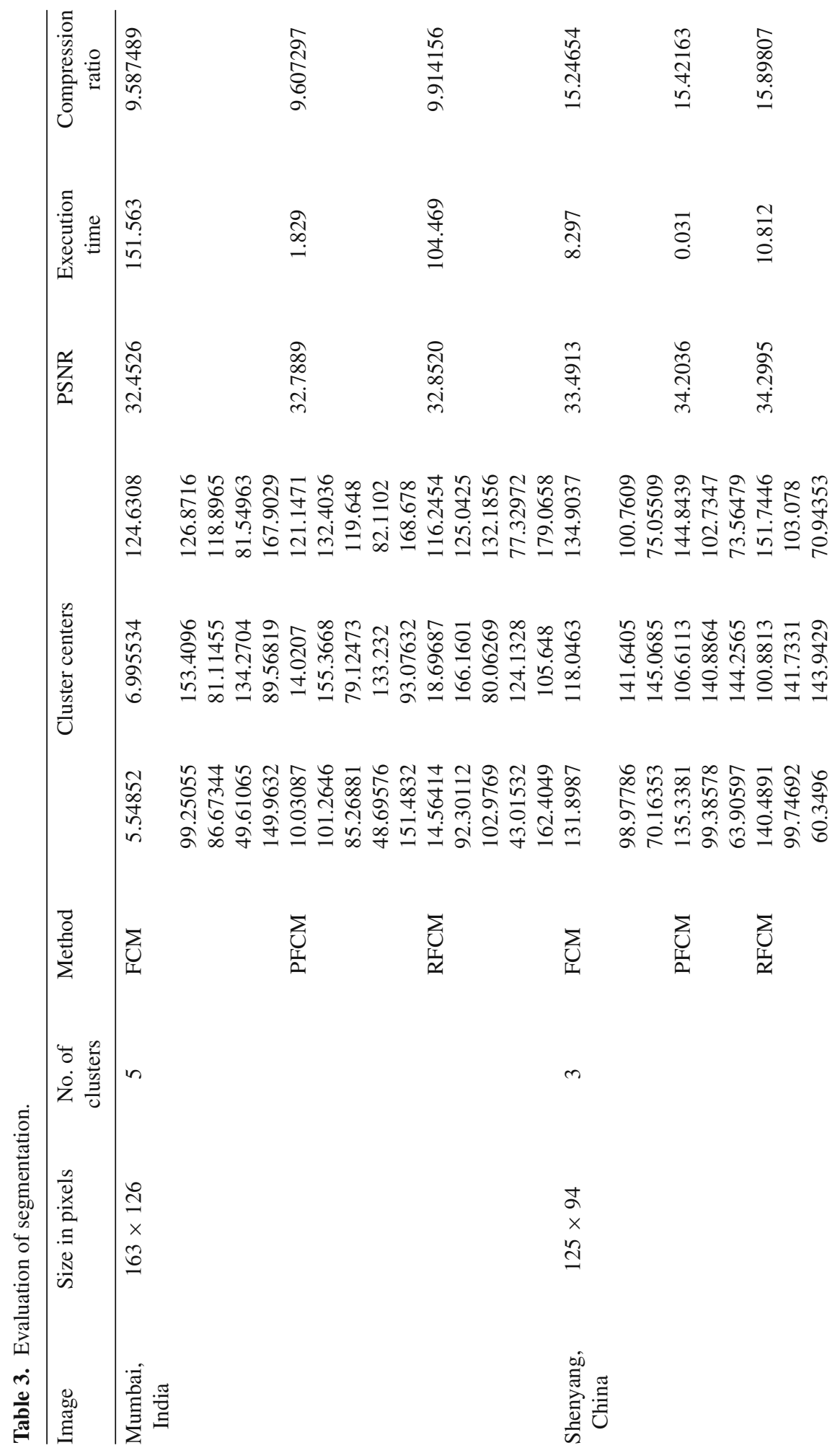




\begin{tabular}{|c|c|c|c|c|}
\hline$\simeq$ & $\simeq$ & $\simeq$ & 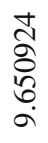 & $\begin{array}{l}\infty \\
\infty \\
\infty \\
\stackrel{9}{+} \\
\infty \\
0\end{array}$ \\
\hline 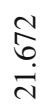 & లై & 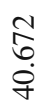 & 于 & $\begin{array}{l}\text { Oे } \\
\stackrel{0}{0}\end{array}$ \\
\hline $\begin{array}{l}m \\
\text { ळे } \\
\dot{m}\end{array}$ & \begin{tabular}{l}
\multirow{+}{+}{} \\
+ \\
$\dot{0}$ \\
$\sim$
\end{tabular} & $\frac{n}{n}$ & $\begin{array}{l}0 \\
\infty \\
2 \\
\dot{n} \\
\infty\end{array}$ & \begin{tabular}{l}
+ \\
\multirow{\delta}{0}{} \\
$\dot{0}$
\end{tabular} \\
\hline
\end{tabular}

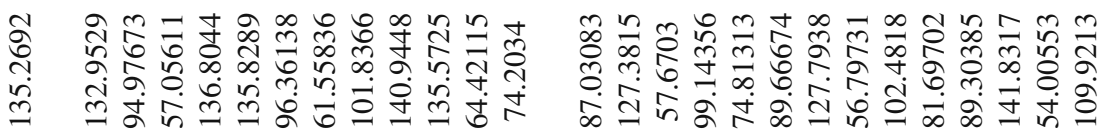

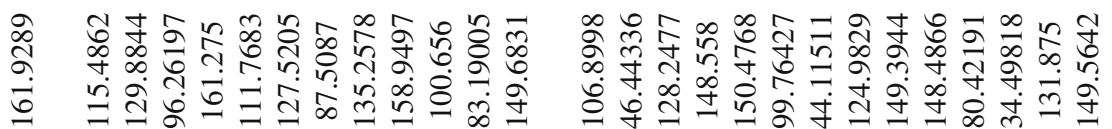

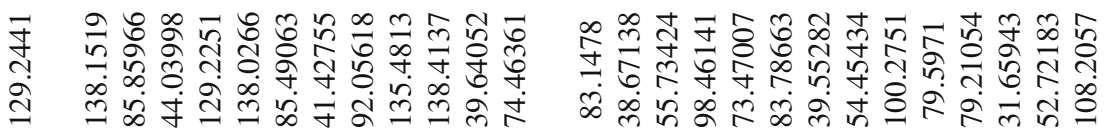

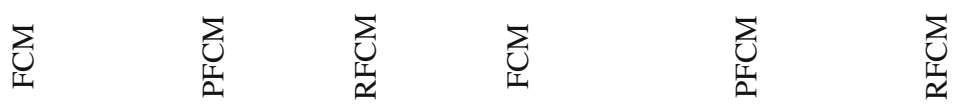

$\forall$

d

$\times$

\&

$\times$

กิ

$\stackrel{2}{\beth}$

兽 


\section{Evaluation of image segmentation and land cover classification}

Four test images of different sizes are used for evaluating the results of the proposed method. They are shown in figure 1 . They are classified using the fuzzy clustering method. The results of classification using FCM is shown in figure 2. Figure 3 shows the classification by PFCM. The land cover classification by RFCM is shown in figure 4.

The evaluation of segmentation by FCM, PFCM and RFCM for the four test images are given in table 3. In the original image pixels are represented by 8 bits. For the segmented image Huffman coding is considered for the calculation of compression ratio.

From the table it can be seen that segmentation by RFCM gives better PSNR for all images. Though the execution time for PFCM is lesser it has to be taken into account that it requires a bootstrap method for initializing its cluster centers and partition matrix.

The execution time taken by RFCM is slightly higher than the FCM. This is expected because of the modification of calculation of partition matrix and the calculation of neighbourhood influence parameter $\gamma$. For the same initial values and termination criterion, i.e., the convergence value of partition matrix, the cluster centers are different for the three algorithms.

The evaluation of classification by FCM, PFCM and RFCM for the four test images are given in table 4. For the calculation of Kappa factor 10 samples of different surface features are considered. For determining Kappa Coefficient for one feature, that particular feature samples are taken as 1 and other feature samples are taken as 0 .

For the Mumbai image, Kappa coefficient of RFCM is high for crops and urban area. PFCM's Kappa coefficient is lowest for urban area. The Forest, wetland vegetation, water samples are correctly classified by all three algorithms. The Kappa coefficient of Sheyang image is same for all three algorithms. The wetland vegetation samples of Zurich image is correctly classified by RFCM whereas the other two algorithms resulted in poor classification. For the Kolkatta image, RFCM results in highest Kappa coefficient for all surface features.

Out of the three methods tested RFCM is found to be good on the basis of image reproduction because of increased PSNR, image compression due to the increased compression ratio as well as accurate classification due to highest Kappa coefficient of all surface features.

Table 4. Evaluation of land cover classification.

\begin{tabular}{|c|c|c|c|c|c|c|c|c|}
\hline \multirow[t]{2}{*}{ Image } & \multirow{2}{*}{$\begin{array}{l}\text { No. of } \\
\text { clusters }\end{array}$} & \multirow[t]{2}{*}{ Method } & \multicolumn{6}{|c|}{ Kappa coefficient of different surface features } \\
\hline & & & Urban area & Bare soil & Forest & Wetland vegetation & Crops & Water \\
\hline \multirow[t]{3}{*}{ Mumbai, India } & \multirow[t]{3}{*}{5} & FCM & 0.8 & - & 1.0 & 1.0 & 0.8 & 1.0 \\
\hline & & PFCM & 0.2 & - & 1.0 & 1.0 & 0.8 & 1.0 \\
\hline & & RFCM & 1.0 & - & 1.0 & 1.0 & 1.0 & 1.0 \\
\hline \multirow[t]{3}{*}{ Shenyang, China } & \multirow[t]{3}{*}{3} & FCM & 0.8 & - & 0.6 & 1.0 & - & - \\
\hline & & PFCM & 0.8 & - & 0.6 & 1.0 & - & - \\
\hline & & RFCM & 0.8 & - & 0.6 & 1.0 & - & - \\
\hline \multirow[t]{3}{*}{ Zurich, Switzerland } & \multirow[t]{3}{*}{4} & FCM & - & 1.0 & 0.8 & 0.8 & 0.8 & 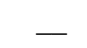 \\
\hline & & PFCM & - & 1.0 & 0.8 & 0.8 & 0.8 & 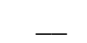 \\
\hline & & RFCM & - & 1.0 & 0.8 & 1.0 & 0.8 & - \\
\hline \multirow[t]{3}{*}{ Kolkatta, India } & \multirow[t]{3}{*}{5} & $\mathrm{FCM}$ & 0.25 & 1.0 & 0.8 & 1.0 & - & 0.8 \\
\hline & & PFCM & 0.25 & 1.0 & 0.6 & 1.0 & - & 0.8 \\
\hline & & RFCM & 0.83 & 1.0 & 0.8 & 1.0 & - & 1.0 \\
\hline
\end{tabular}




\section{Conclusion}

A new reformed fuzzy $\mathrm{C}$ means technique has been proposed for land cover classification. The satellite image segmentation and land cover classification by RFCM are compared with that of FCM and PFCM on the quality measures PSNR, compression ratio, execution time and Kappa coefficient. From the quality measures it can be seen that RFCM is better than the other fuzzy clustering techniques. The following points are worth stressing.

(i) The image segmentation by RFCM gives best PSNR. Hence, it is well-suited for image reproduction.

(ii) The compression ratio obtained by RFCM is the Highest. So, it is quite useful for data compression.

(iii) The convergence time of the PFCM is the lowest. But it requires a bootstrap method for cluster centers and partition matrix initialization.

(iv) The Kappa coefficient for all surface features is highest for RFCM. It is very useful for land cover classification.

The program developed has been tested with various pictures and the results were proven to be fruitful. The program has also been tested for its consistency and its reliability. This work is useful for land cover mapping. Land cover maps are essential inputs to environmental and land use planning at local, regional, and national levels. They are being used directly in resource inventories like woodland census, state of the environment reporting like establishing the distribution, extent and quality of key habitats. They also act as input to the development and monitoring of a range of land use and conservation policies.

\section{References}

Bastin L 1997 Comparison of fuzzy C-means classification, linear mixture modeling and MLC probabilities as tools for unmixing coarse pixels. International J. Remote Sensing 18(17): 3629-3648

Bezdek J C 1981 Pattern recognition with fuzzy objective function algorithms. NY: Plenum Press

Cox E 2005 Fuzzy modeling and genetic algorithms for data mining and exploration. United States of America: Elsevier

Dunn J C 1973 A fuzzy relative of the ISODATA process and its use in detecting compact well-separated clusters. J. Cybernetics 3: 32-57

Foody G M 1996 Approaches for the production and evaluation of fuzzy land cover classifications from remotely-sensed data. Int. J. Remote Sensing 17(7): 1317-1340

Foody G M, Zhang J 1998 A fuzzy classification of sub-urban land cover from remotely sensed imagery. Int. J. Remote Sensing 19(14): 2721-2738

Frank K, Annette K 1997 Fuzzy clustering based on modified distance measures. Proceedings of the third international symposium on advances in intelligent data analysis, Aug 01, 291-302

Ibrahim M A, Arora M K, Ghosh S K 2005 Estimating and accommodating uncertainty through the soft classification of remote sensing data. Int. J. of Remote Sensing 26(14): 2995-3007

Krishnapuram R, Keller J M 1996 The possibilistic C-means algorithm: Insights and recommendations. IEEE Transactions on Fuzzy Systems 4(3): 385-393

Lei J, Wenhui Y 2003 A modified fuzzy C-means algorithm for segmentation of magnetic resonance images. Proc. VIIth digital image computing: Techniques and applications, Sydney, 10-12 Dec., 225-231

Pal N R, Pal K, Keller J M, Bezdek J C 2005 A possibilistic fuzzy C-means clustering algorithm. IEEE Transactions on Fuzzy Systems 13(4): 517-530

Wang F 1990 Fuzzy supervised classification of remote sensing images. IEEE Trans. Geosci. Remote Sens. 28(2): 194-201 